\title{
Partial Relay Selection With Outdated Channel State Estimation in Mixed RF/FSO Systems
}

\author{
Milica I. Petkovic, Student Member, IEEE, Aleksandra M. Cvetkovic, Student Member, IEEE, \\ Goran T. Djordjevic, Member, IEEE, and George K. Karagiannidis, Fellow, IEEE
}

\begin{abstract}
We study a dual-hop relaying system, composed of mixed radio frequency (RF)/free-space optical (FSO) links. Partial amplify-and-forward relay selection is assumed, which is based on outdated channel state information (CSI). The RF link is subject to Rayleigh fading, while the FSO link is affected by atmospheric turbulence, modeled by Gamma-Gamma distribution. Novel analytical expressions for the outage probability and average bit error rate (BER) are derived. Numerical results are presented and validated by Monte-Carlo simulations. The outage probability and average BER dependence on turbulence strength, average signalto-noise ratio (SNR) over RF hop, average electrical SNR over FSO hop, number of relays, and rank of selected relay, are also investigated, while the effect of time correlation between current source-relay channel and its outdated CSI, is considered. The results illustrate that the outdated CSI used for the relay selection has a strong effect on the system performance, especially in weak turbulence conditions of the FSO hop. Furthermore, the improvement of the system performance with an increase of the number of the relays is highly dependent on the FSO channel state.
\end{abstract}

Index Terms-Bit error rate, free space optical systems, outage probability, outdated channel state information, partial relay selection, radio frequency systems.

\section{INTRODUCTION}

$\mathbf{M}$ IXED dual-hop amplify-and-forward (AF) relaying systems composed of radio-frequency (RF) and free-space optical (FSO) links were primarily studied in [1]. The difficulty and expense of deploying fiber-optics have been the reason to employ FSO as a last mile solution, proposing license-free operation, non-interfering nature, simple and low-cost implementation and high data rate capacity. On the other hand, relay systems offer coverage area extensions and better system performance at the long distance transmission. Due to the aforementioned benefits of both relaying technology and FSO as the last mile

Manuscript received September 17, 2014; revised March 6, 2015; accepted March 11, 2015. Date of publication March 26, 2015; date of current version May 22, 2015. This work was supported by the European Science Foundation under COST IC1101 OPTICWISE Action "Optical Wireless Communications-An Emerging Technology," Ministry of Education, Science, and Technology Development of the Republic of Serbia under Grants TR-32028 and III-44006, and Ministry of the Foreign Affairs of Norway within the NORBAS Project.

M. I. Petkovic, A. M. Cvetkovic, and G. T. Djordjevic are with the Faculty of Electronic Engineering, University of Nis, 18000 Nis, Serbia (e-mail milica.petkovic@elfak.ni.ac.rs; aleksandra.cvetkovic@elfak.ni.ac.rs; goran@ elfak.ni.ac.rs)

G. K. Karagiannidis is with the Aristotle University of Thessaloniki, Thessaloniki 54124, Greece, and also with the Khalifa University, Abu Dhabi, UAE (e-mail: geokarag@auth.gr).

Color versions of one or more of the figures in this paper are available online at http://ieeexplore.iee.org.

Digital Object Identifier 10.1109/JLT.2015.2416972 solution, the interest in RF/FSO relaying systems has been in constant progression [2]-[7].

The outage performance of mixed dual-hop AF RF/FSO relaying system in various turbulence conditions was presented in [1] and [2], while analysis in [3]-[7] has been extended to amount of fading, bit error rate (BER), symbol error rate and ergodic capacity. In all papers mentioned above, a single relay was assumed for the RF/FSO system. In multiple relay systems, further improvement of system performance can be achieved using some relay selection schemes [8]. Contrary to the best and opportunistic relay selections, which require channel state information (CSI) of each hop, Krikidis et al. proposed partial relay selection (PRS) in [9]. This scheme assumes that the relay is selected according to CSI of only one hop (either source-relay or relay-destination link), so additional power waste and further network delays will be avoided.

However, in time-varying channels the instantaneous CSI used for relay selection can be outdated due to feedback delay, so the selected relay is not necessarily the best in transmission slot. This results in degradation of system performance, which depends on time-correlation coefficient. In contrast to [9]-[11], where PRS scheme is considered with perfect CSI, the feedback delay effect over Rayleigh and Nakagami- $m$ fading channels, were investigated in [12]-[14] and [15], respectively. In the case when the best relay cannot be able to forward the signal, the selected relay will be the best among the set of remaining relays, i.e., $k$ th worst relay will be selected, as was proposed in [12].

To the best of the authors' knowledge, the mixed dual-hop RF/FSO systems with PRS have not been investigated yet. In addition, the effects of outdated estimation on the systems with FSO links have not been investigated so far. In this paper, we analyse dual-hop AF RF/FSO systems with relay selection procedure based on outdated CSI. It is considered that the RF link is subject to Rayleigh fading [16], while FSO link experiences Gamma-Gamma fading, applicable in a wide range of turbulence conditions [17]-[19]. Subcarrier intensity modulation (SIM) is used for electrical-optical signal conversion at the relay [18]. Novel analytical expressions for outage probability and average BER are derived and reduced to the special case of PRS with perfect CSI. Also, if only one relay is assumed, then outage probability and average BER expressions are reduced to the corresponding ones, reported in [1] and [4], respectively. Monte-Carlo simulations are used to validate the derived analytical results.

The rest of the paper is organized as follows. Section II describes the system and channel model. The outage probability 


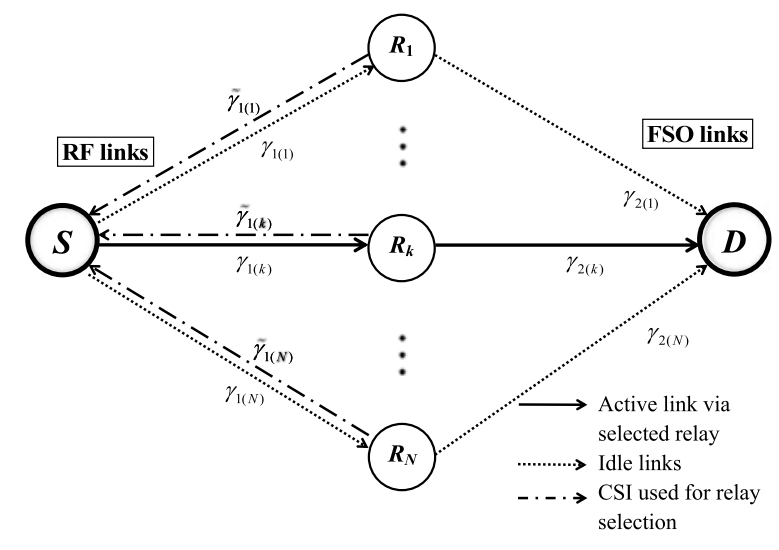

Fig. 1. A mixed RF/FSO system with PRS.

analysis is presented in Section III, while Section IV contains average BER analysis. Numerical results with discussions are presented in Section V, while some concluding remarks are given in Section VI.

\section{System AND ChANNEL Model}

Mixed dual-hop RF/FSO systems have been proposed in order to exploit FSO benefits as a last mile solution, when direct lineof-sight (LOS) component between transmitter and receiver is not available. Relaying technology enables the bridging of nonLOS area by RF link, while FSO link distance is reduced resulting in optical power saving. The RF/FSO relaying systems are proposed to overcome connectivity gap between a backbone and a last mile network (end users). They are valuable for enabling a large number of RF users to be multiplexed through a single FSO link. The similar scenario was observed in [2]-[4] and [6]. Multiple relay systems with different relay selection schemes have been introduced for achieving a large diversity gain. Fig. 1 presents a mixed AF dual-hop RF/FSO system with PRS, which consists of a source $S$, a destination $D$ and $N \geq 1$ relays. Node $S$ supervises the conditions of RF Rayleigh fading channel via local feedback from the relays. According to the instantaneous CSI estimation, the node $S$ selects the relay for further transmission in the following way: the instantaneous signal-to-noise ratios (SNRs), $\tilde{\gamma}_{1(l)}$ for $l=1, \ldots, N$, obtained by channel estimations, are arranged in an increasing order of magnitude as $\tilde{\gamma}_{1(1)} \leq \tilde{\gamma}_{1(2)} \leq \cdots \leq \tilde{\gamma}_{1(N)}$. According to the ideal scenario, node $S$ selects the best relay [9]-[11]. However, the selected relay may not be available, so source will choose the next best relay etc. In other words, the PRS is performed by selecting the $k$ th worst or $(N-k)$ th best relay $R_{(k)}$ [12]. Furthermore, in practice, the current CSI of the RF channel is not the same as the CSI used for relay selection, so PRS with outdated CSI is assumed. Therefore, the instantaneous SNR of RF link, $\gamma_{1(k)}$, and the instantaneous SNR used for PRS, $\tilde{\gamma}_{1(k)}$, are two correlated random variables with correlation coefficient $\rho$.

The received electrical signal at the $k$ th relay is

$$
r_{R(k)}(t)=h_{S R(k)}(t) r(t)+n_{S R}(t),
$$

where $r(t)$ is the RF signal, transmitted from $S$ with an average power $P_{S}, h_{S R(k)}(t)$ is the fading amplitude of the $S-R_{(k)}$ link. The additive white Gaussian noise (AWGN) in RF link, with zero mean and variance $\sigma_{S R}^{2}$, is denoted as $n_{S R}(t)$.

Next, it is assumed that the received signal at node $R_{(k)}$ is amplified by a proper relay gain $G$. Fixed gain relay is assumed, so the amplification is performed based on the statistical CSI (also called semi-blind). The relay gain $G$ is [13, eq. (3)]

$$
\begin{aligned}
G^{2} & =\frac{P_{R}}{\mathrm{E}\left[\left|h_{S R(k)}(t)\right|^{2}\right] P_{S}+\sigma_{S R}^{2}} \\
& =\frac{P_{R}}{\sigma_{S R}^{2}} \frac{1}{\mathrm{E}\left[\gamma_{1(k)}\right]+1}=\frac{P_{R}}{\sigma_{S R}^{2} C},
\end{aligned}
$$

where $C$ is a constant determined by the relay gain, $P_{R}$ is the relay output signal power and $\mathrm{E}[\cdot]$ denotes expectation. The instantaneous SNR of the first RF hop is

$$
\gamma_{1(k)}=\frac{\left|h_{S R(k)}(t)\right|^{2} P_{S}}{\sigma_{S R}^{2}}=\left|h_{S R(k)}(t)\right|^{2} \mu_{1},
$$

where $\mu_{1}=P_{S} / \sigma_{S R}^{2}$ is the average SNR. The FSO part of the system employs intensity modulation with direct detection, while a subcarrier signal is used to modulate intensity of an optical carrier (representing SIM technique). The RF signal is converted to an optical one, which is given by [1, eq. (2)], [2, eq. (3)]

$$
r_{\text {opt }}(t)=G\left(1+\eta_{e} r_{R(k)}(t)\right),
$$

with $\eta_{e}$ being the electrical-to-optical conversion coefficient. It is assumed that $\eta_{e}=1$, so it will be neglected in the rest of the paper.

At the reception, direct detection is performed by removing dc bias and carrying out optical to electrical conversion, and the signal at node $D$ is expressed as

$$
r_{D(k)}(t)=I_{R(k) D}(t) G \eta r_{R(k)}(t)+n_{R D}(t),
$$

where $I_{R(k) D}(t)$ is the intensity of an optical signal, $\eta$ is an optical-to-electrical conversion coefficient and $n_{R D}(t)$ is AWGN over FSO link, with zero mean and variance $\sigma_{R D}^{2}$.

The overall SNR at the destination can be obtained as [11, eq. (1)]

$$
\gamma_{e q}=\frac{\gamma_{2(k)} \gamma_{1(k)}}{\gamma_{2(k)}+C}
$$

where

$$
\gamma_{2(k)}=\frac{\left|I_{R(k) D}(t)\right|^{2} \eta^{2} P_{R}}{\sigma_{R D}^{2}}=\left|I_{R(k) D}(t)\right|^{2} \mu_{2},
$$

is the instantaneous electrical SNR over FSO link, which experiences Gamma-Gamma fading, with average electrical SNR, $\mu_{2}=\eta^{2} P_{R} / \sigma_{R D}^{2}$. The average electrical SNR, $\mu_{2}$, is related to average SNR, $\bar{\gamma}_{2}$, by $\mu_{2}=\bar{\gamma}_{2} \alpha \beta /((\alpha+1)(\beta+1))$ [20, eq. (8)].

Since RF link operates in Rayleigh fading channel, following the derivations of Appendix A, the probability density function 
(PDF) of the instantaneous SNR in the first hop is [12, eq. (9)]

$$
\begin{aligned}
& f_{\gamma_{1(k)}}(x)=k\left(\begin{array}{l}
N \\
k
\end{array}\right) \sum_{n=0}^{k-1}\left(\begin{array}{c}
k-1 \\
n
\end{array}\right) \frac{(-1)^{n}}{\mu_{1}} \\
& \times \frac{1}{(N-k+n)(1-\rho)+1} e^{-\frac{(N-k+n+1) x}{((N-k+n)(1-\rho)+1) \mu_{1}}} .
\end{aligned}
$$

The cumulative distribution function (CDF) of $\gamma_{1(k)}$ can be obtained as

$$
\begin{aligned}
F_{\gamma_{1(k)}}(x)= & \int_{0}^{x} f_{\gamma_{1(k)}}(t) d t=1-k\left(\begin{array}{c}
N \\
k
\end{array}\right) \sum_{n=0}^{k-1}\left(\begin{array}{c}
k-1 \\
n
\end{array}\right) \\
& \times \frac{(-1)^{n}}{(N-k+n+1)} e^{-\frac{(N-k+n+1) x}{((N-k+n)(1-\rho)+1) \mu_{1}}}
\end{aligned}
$$

while the constant $C=\mathrm{E}\left[\gamma_{1(k)}\right]+1$ is found using (2) and (8) as [13, eq. (6)]

$$
\begin{aligned}
C= & 1+\int_{0}^{\infty} x f_{\gamma_{1(k)}}(x) d x=1+k\left(\begin{array}{c}
N \\
k
\end{array}\right) \sum_{n=0}^{k-1}\left(\begin{array}{c}
k-1 \\
n
\end{array}\right) \\
& \times \frac{(-1)^{n}((N-k+n)(1-\rho)+1) \mu_{1}}{(N-k+n+1)^{2}}
\end{aligned}
$$

The FSO hop is under the influence of atmospheric turbulence, and the intensity fluctuations at the received signal are assumed to follow the Gamma-Gamma distribution. So, the instantaneous electrical SNR, $\gamma_{2(k)}$, has a PDF given by [1, eq. (8)]

$$
f_{\gamma_{2(k)}}(x)=\frac{(\alpha \beta)^{\frac{\alpha+\beta}{2}} x^{\frac{\alpha+\beta}{4}-1}}{\Gamma(\alpha) \Gamma(\beta) \mu_{2}^{\frac{\alpha+\beta}{4}}} K_{\alpha-\beta}\left(2 \sqrt{\alpha \beta \sqrt{\frac{x}{\mu_{2}}}}\right),
$$

where $K_{\nu}(\cdot)$ is the $\nu$ th order modified Bessel function of the second kind [21, eq. (8.432.2)]. The parameters $\alpha$ and $\beta$ are the effective numbers of small-scale and large-scale eddies of the scattering environment, respectively, related to the atmospheric conditions. If plane wave propagation and zero inner scale is assumed, the parameters $\alpha$ and $\beta$ are [17, p. 511, eq. (68)], [18, p. 139, eqs. (3.127), (3.128)]

$$
\begin{aligned}
& \alpha=\left(\exp \left[\frac{0.49 \sigma_{R}^{2}}{\left(1+1.11 \sigma_{R}^{12 / 5}\right)^{7 / 6}}\right]-1\right)^{-1}, \\
& \beta=\left(\exp \left[\frac{0.51 \sigma_{R}^{2}}{\left(1+0.69 \sigma_{R}^{12 / 5}\right)^{5 / 6}}\right]-1\right)^{-1},
\end{aligned}
$$

where the Rytov variance $\sigma_{R}^{2}$ is used as a metric of the turbulence strength.

It is well known that atmospheric turbulence over FSO link results in slowly-varying fading. Since the coherence time of the channel is about $1-100 \mathrm{~ms}$, turbulence induced fading remains constant over a large number of transmitted bits [22]-[24]. In addition, the use of very long interleavers in order to achieve independence of fading samples in consecutive symbol intervals is not practical in the FSO channel [23]. However, our aim is to examine the best possible average BER performance, or in other words, to determine upper bound of performance. Hence, our average BER analysis is valid under the assumption that the information symbol is long enough to ensure long-term ergodic properties of the turbulence process [22]. Besides average BER, we are also interested in outage probability indicating how often the system is under the desired performance threshold.

\section{Outage Probability ANALysis}

The outage probability is defined as the probability that the instantaneous end-to-end SNR falls below a predetermined outage threshold $\gamma_{t h}$. In the system under consideration, the outage probability can be written as

$$
P_{\text {out }}=F_{\text {eq }}\left(\gamma_{\text {th }}\right)=\operatorname{Pr}\left(\frac{\gamma_{2(k)} \gamma_{1(k)}}{\gamma_{2(k)}+C}<\gamma_{t h} \mid \gamma_{2(k)}\right),
$$

where $\operatorname{Pr}(\cdot)$ denotes probability. After some mathematical manipulations, (13) can be written as

$$
\begin{aligned}
P_{\text {out }} & =\int_{0}^{\infty} \operatorname{Pr}\left(\gamma_{1(k)}<\gamma_{t h}+\frac{\gamma_{t h} C}{\gamma_{2(k)}}\right) f_{\gamma_{2(k)}}\left(\gamma_{2(k)}\right) d \gamma_{2(k)} \\
& =\int_{0}^{\infty} F_{\gamma_{1(k)}}\left(\gamma_{t h}+\frac{\gamma_{t h} C}{x}\right) f_{\gamma_{2(k)}}(x) d x
\end{aligned}
$$

Substituting (9) and (11) into (14), and after some mathematical derivations presented in Appendix B, the outage probability can be expressed in terms of Meijer's $G$ function [21, eq. (9.301)] as

$$
\begin{aligned}
P_{\text {out }}= & 1-k\left(\begin{array}{c}
N \\
k
\end{array}\right) \sum_{n=0}^{k-1}\left(\begin{array}{c}
k-1 \\
n
\end{array}\right) \frac{(-1)^{n}}{(N-k+n+1)} \\
& \times \frac{2^{\alpha+\beta-2}}{\pi \Gamma(\alpha) \Gamma(\beta)} e^{-\frac{(N-k+n+1) \gamma_{h}}{((N-k+n)(1-\rho)+1) \mu_{1}}} \\
& \times G_{0,5}^{5,0}\left(\frac{\alpha^{2} \beta^{2} \gamma_{h t} C(N-k+n+1)}{16 \mu_{2} \mu_{1}((N-k+n)(1-\rho)+1)} \mid \begin{array}{c}
- \\
\kappa_{1}
\end{array}\right)
\end{aligned}
$$

where

$$
\kappa_{1}=\frac{\alpha}{2}, \quad \frac{\alpha+1}{2}, \quad \frac{\beta}{2}, \quad \frac{\beta+1}{2}, \quad 0 .
$$

\section{A. Special Cases: Perfect and Completely Outdated CSI}

Assuming PRS with perfect CSI, i.e., instantaneous CSI used for relay selection is fully correlated with the current CSI of the RF channel, the outage probability can be obtained by substituting $\rho=1$ into (15) as

$$
\begin{aligned}
P_{\text {out }}^{\rho=1}= & 1-k\left(\begin{array}{c}
N \\
k
\end{array}\right) \sum_{n=0}^{k-1}\left(\begin{array}{c}
k-1 \\
n
\end{array}\right) \frac{(-1)^{n}}{(N-k+n+1)} \\
& \times \frac{2^{\alpha+\beta-2}}{\pi \Gamma(\alpha) \Gamma(\beta)} e^{-\frac{(N-k+n+1) \gamma_{t h}}{\mu_{1}}} \\
& \times G_{0,5}^{5,0}\left(\frac{\alpha^{2} \beta^{2} \gamma_{t h} C_{\rho=1}(N-k+n+1)}{16 \mu_{2} \mu_{1}} \mid \begin{array}{l}
- \\
\kappa_{1}
\end{array}\right),
\end{aligned}
$$


while the constant $C_{\rho=1}$ is obtained by substituting $\rho=1$ into (10) as

$$
C_{\rho=1}=1+k\left(\begin{array}{c}
N \\
k
\end{array}\right) \sum_{n=0}^{k-1}\left(\begin{array}{c}
k-1 \\
n
\end{array}\right) \frac{(-1)^{n} \mu_{1}}{(N-k+n+1)^{2}} .
$$

When PRS is based on completely outdated CSI, i.e., instantaneous CSI used for relay selection is totally outdated with the current CSI of the RF channel, the outage probability can be found by substituting $\rho=0$ into (15) as

$$
\begin{aligned}
P_{\text {out }}^{\rho=0}= & 1-k\left(\begin{array}{c}
N \\
k
\end{array}\right) \sum_{n=0}^{k-1}\left(\begin{array}{c}
k-1 \\
n
\end{array}\right) \frac{(-1)^{n}}{(N-k+n+1)} \\
& \times \frac{2^{\alpha+\beta-2}}{\pi \Gamma(\alpha) \Gamma(\beta)} e^{-\frac{\gamma_{t h}}{\mu_{1}}} G_{0,5}^{5,0}\left(\frac{\alpha^{2} \beta^{2} \gamma_{t h} C_{\rho=0}}{16 \mu_{2} \mu_{1}} \mid \begin{array}{c}
- \\
\kappa_{1}
\end{array}\right),
\end{aligned}
$$

with the constant $C_{\rho=0}$ derived by substituting $\rho=0$ into (10) as

$$
C_{\rho=0}=1+k\left(\begin{array}{c}
N \\
k
\end{array}\right) \sum_{n=0}^{k-1}\left(\begin{array}{c}
k-1 \\
n
\end{array}\right) \frac{(-1)^{n} \mu_{1}}{(N-k+n+1)} .
$$

\section{B. Ideal Relay Selection}

Ideal PRS implies that the selected relay is one with the best estimated instantaneous SNR, i.e., $k=N$, and that it is available for further transmission. Applying $k=N$ in (15), the outage probability is given by

$$
\begin{aligned}
& P_{\text {out }}^{k=N}=1-N \sum_{n=0}^{N-1}\left(\begin{array}{c}
N-1 \\
n
\end{array}\right) \frac{(-1)^{n}}{(n+1)} \frac{2^{\alpha+\beta-2}}{\pi \Gamma(\alpha) \Gamma(\beta)} \\
& \quad \times e^{-\frac{(n+1) \gamma_{t h}}{((1-\rho) n+1) \mu_{1}}} G_{0,5}^{5,0}\left(\frac{\alpha^{2} \beta^{2} \gamma_{t h} C_{k=N}(n+1)}{16 \mu_{2} \mu_{1}((1-\rho) n+1)} \mid \begin{array}{c}
- \\
\kappa_{1}
\end{array}\right),
\end{aligned}
$$

where the constant $C_{k=N}$ is

$C_{k=N}=1+N \sum_{n=0}^{N-1}\left(\begin{array}{c}N-1 \\ n\end{array}\right) \frac{(-1)^{n}((1-\rho) n+1) \mu_{1}}{(n+1)^{2}}$.

Note, that as above, the two extreme correlated cases when ideal PRS is performed can be evaluated by substituting $\rho=1$ or $\rho=0$ into (21).

\section{System With a Single Relay}

If the system under the consideration consists of one relay, substituting $N=k=1$ into (15) leads to the outage probability for the RF/FSO system without PRS, written as

$P_{\text {out }}^{N=1}=1-\frac{2^{\alpha+\beta-2}}{\pi \Gamma(\alpha) \Gamma(\beta)} e^{-\frac{\gamma_{t h}}{\mu_{1}}} G_{0,5}^{5,0}\left(\left.\frac{\alpha^{2} \beta^{2} \gamma_{t h} C_{N=1}}{16 \mu_{2} \mu_{1}}\right|_{\kappa_{1}} ^{-}\right)$,

where the constant $C_{N=1}$ is obtained by substituting $N=1$ into (10) as

$$
C_{N=1}=1+\mu_{1} .
$$

Using [25, eq. (07.34.16.0001.01)], (23) reduces to [1, eq. (10)].

\section{AVERAGE BER ANALYSIS}

The average BER expressions are derived in the case when binary phase-shift keying (BPSK) [16], is applied over RF link and SIM BPSK [18], is applied over FSO link, as well as in the case of application of differential BPSK (DBPSK) [16], over RF link and SIM DBPSK [18], over FSO link. These two combinations of modulation schemes are denoted by scheme 1 and scheme 2, respectively. The average BER can be derived following the approach presented in [3] and [26]. Specifically, for the RF/FSO system under PRS with feedback delay, the average BER can be found using [26, eq. (12)] as

$$
P_{b}=\frac{q^{p}}{2 \Gamma(p)} \int_{0}^{\infty} e^{-q \gamma_{t h}} F_{e q}\left(\gamma_{t h}\right) d \gamma_{t h}
$$

where the parameters $p$ and $q$ account for different modulation schemes: $(p, q)=(0.5,1)$ for scheme $1 ;(p, q)=(1,1)$ for scheme 2 [3].

After substituting (15) into (25) and utilizing [25, eqs. (01.03.26.0004.01) and (07.34.21.0011.01)], the average BER is derived after some mathematical manipulations as

$$
\begin{aligned}
P_{b}= & \frac{1}{2}-k\left(\begin{array}{c}
N \\
k
\end{array}\right) \sum_{n=0}^{k-1}\left(\begin{array}{c}
k-1 \\
n
\end{array}\right) \frac{(-1)^{n}}{(N-k+n+1)} \\
& \times \frac{2^{\alpha+\beta-3}}{\pi \Gamma(p) \Gamma(\alpha) \Gamma(\beta)}\left(1+\frac{(N-k+n+1)}{q((N-k+n)(1-\rho)+1) \mu_{1}}\right)^{-p} \\
& \times G_{1,5}^{5,1}\left(\frac{\alpha^{2} \beta^{2} C}{16 \mu_{2}\left(q \mu_{1} \frac{((N-k+n)(1-\rho)+1)}{N-k+n+1}+1\right)} \mid \begin{array}{c}
1-p \\
\kappa_{1}
\end{array}\right) .
\end{aligned}
$$

\section{A. Special Cases: Perfect and Completely Outdated CSI}

The average BER for PRS with perfect CSI is obtained by substituting $\rho=1$ into (26) as

$$
\begin{aligned}
P_{b}^{\rho=1}= & \frac{1}{2}-k\left(\begin{array}{c}
N \\
k
\end{array}\right) \sum_{n=0}^{k-1}\left(\begin{array}{c}
k-1 \\
n
\end{array}\right) \frac{(-1)^{n}}{(N-k+n+1)} \\
& \times \frac{2^{\alpha+\beta-3}}{\pi \Gamma(p) \Gamma(\alpha) \Gamma(\beta)}\left(1+\frac{(N-k+n+1)}{q \mu_{1}}\right)^{-p} \\
& \times G_{1,5}^{5,1}\left(\frac{\alpha^{2} \beta^{2} C_{\rho=1}}{16 \mu_{2}\left(\frac{q \mu_{1}}{N-k+n+1}+1\right)} \mid \begin{array}{c}
1-p \\
\kappa_{1}
\end{array}\right)
\end{aligned}
$$

Assuming that PRS is based on completely outdated CSI, the average BER is found by substituting $\rho=0$ into (26) as

$$
\begin{aligned}
P_{b}^{\rho=0}= & \frac{1}{2}-k\left(\begin{array}{c}
N \\
k
\end{array}\right) \sum_{n=0}^{k-1}\left(\begin{array}{c}
k-1 \\
n
\end{array}\right) \frac{(-1)^{n}}{(N-k+n+1)} \\
& \times \frac{2^{\alpha+\beta-3}}{\pi \Gamma(p) \Gamma(\alpha) \Gamma(\beta)}\left(1+\frac{1}{q \mu_{1}}\right)^{-p} \\
& \times G_{1,5}^{5,1}\left(\frac{\alpha^{2} \beta^{2} C_{\rho=0}}{16 \mu_{2}\left(q \mu_{1}+1\right)} \mid \begin{array}{c}
1-p \\
\kappa_{1}
\end{array}\right) .
\end{aligned}
$$




\section{B. Ideal Relay Selection}

When relay with the best estimated instantaneous SNR is available for further transmission, the average BER is obtained by substituting $k=N$ into (26) as

$$
\begin{aligned}
P_{b}^{k=N}= & \frac{1}{2}-N \sum_{n=0}^{N-1}\left(\begin{array}{c}
N-1 \\
n
\end{array}\right) \frac{(-1)^{n}}{(n+1)} \\
& \times \frac{2^{\alpha+\beta-3}}{\pi \Gamma(p) \Gamma(\alpha) \Gamma(\beta)}\left(1+\frac{n+1}{((1-\rho) n+1) q \mu_{1}}\right)^{-p} \\
& \times G_{1,5}^{5,1}\left(\frac{\alpha^{2} \beta^{2} C_{k=N}}{16 \mu_{2}\left(q \mu_{1} \frac{(1-\rho) n+1}{n+1}+1\right)} \mid \begin{array}{c}
1-p \\
\kappa_{1}
\end{array}\right) \cdot
\end{aligned}
$$

By applying $\rho=1$ or $\rho=0$ into (29), the two correlated cases for ideal PRS can be obtained.

\section{System With a Single Relay}

Substituting $N=k=1$ into (26), the average BER for mixed $\mathrm{RF} / \mathrm{FSO}$ system with single relay is obtained as

$$
\begin{aligned}
P_{b}^{N=1}= & \frac{1}{2}-\frac{2^{\alpha+\beta-3}}{\pi \Gamma(p) \Gamma(\alpha) \Gamma(\beta)}\left(1+\frac{1}{q \mu_{1}}\right)^{-p} \\
& \times G_{1,5}^{5,1}\left(\frac{\alpha^{2} \beta^{2} C_{N=1}}{16 \mu_{2}\left(q \mu_{1}+1\right)} \mid \begin{array}{c}
1-p \\
\kappa_{1}
\end{array}\right),
\end{aligned}
$$

which is previously derived and presented in [4, eq. (15)]. After applying [25, eq. (07.34.16.0001.01)], the average BER in (30) comes to [3, eq. (17)].

\section{NUMERICAL RESULTS}

This section presents numerical examples based on the analytical results reported in this paper. The outage probability and average BER dependence on different parameters are investigated and discussed. Numerical results are validated by Monte-Carlo simulations.

The outage probability dependence on Rytov standard deviation $\sigma_{R}$ is presented in Fig. 2. The system when the best relay is able to forward the signal $(k=N)$ and the system when all except the worst relay are unavailable $(k=1)$ are observed for various correlation coefficients. As it was expected, the system performs better when the best relay is selected. In that case, an increase in the correlation coefficient leads to lower outage probability. Assuming perfect PRS $(\rho=1)$, the full correlation between CSI used for decision of relay selection and actual RF channel state is achieved, which is manifested in the best system performance. However, if relay selection is based on the worst relay, the outage performance will deteriorate with an increase of $\rho$. In that case, the outdated CSI used for relay selection is the less adequate for transmission. When $\rho=1$, actual and outdated CSI are the same indicating the chosen relay is probably the worst. On the other hand, when $\rho=0$, actual and outdated CSI are completely uncorrelated, and it can be concluded with high probability that the chosen relay is not the worst, leading to the best system performance.

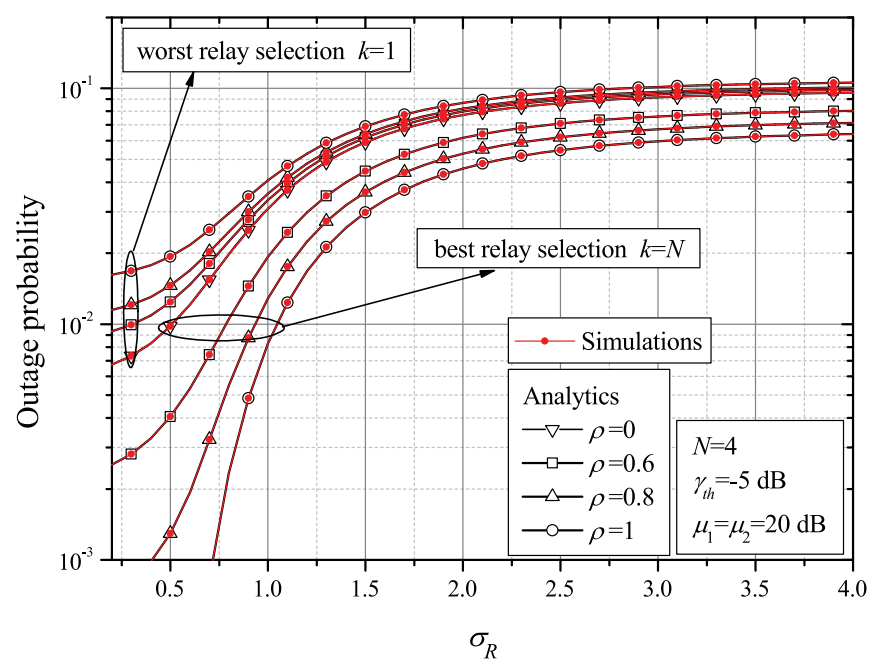

Fig. 2. Outage probability versus $\sigma_{R}$ for different values of correlation coefficient $\rho$.

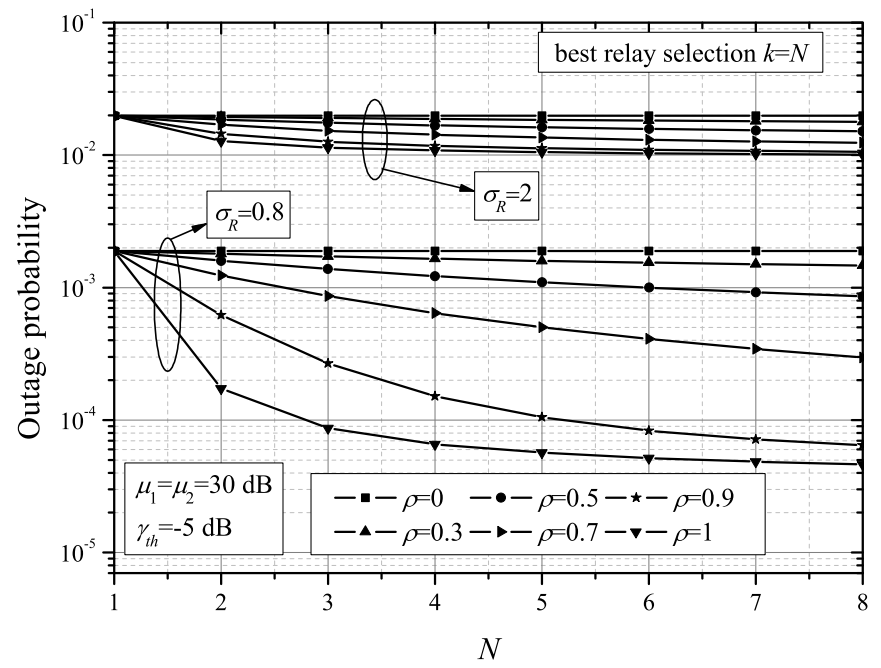

Fig. 3. Outage probability versus $N$ for different values of $\rho$ in different turbulence conditions.

When completely outdated PRS is assumed $(\rho=0)$, the outdated CSI used for relay selection and actual first hop CSI are completely independent and the choice of relay is of no importance to the system performance. Therefore, the overlapping of curves in Fig. 2. for $(k=N)$ and $(k=1)$ is observed.

Furthermore, the influence of the correlation coefficient is greater in weak conditions $\left(\sigma_{R}<2\right)$, compared to moderate $\left(\sigma_{R}=2\right)$ and strong $\left(\sigma_{R}>2\right)$ ones. When FSO link is affected by weak turbulence, which corresponds to clear air terms, there will be no severe attenuation at the second hop. Hence, the state of the RF part of the system will be crucial and diversity of PRS decision and correlation coefficient have major impact on the outage performance. On the other hand, in the strong turbulence conditions, the optical signal will be degraded to a large extent, which results in low correlation impact.

The outage probability dependence on number of relays for different values of correlation coefficient is presented in Fig. 3. Two cases are observed: weak and moderate turbulence 
TABLE I

OutAge Probability For DifFERENT NUMBER of RELAYS $N$

\begin{tabular}{lcccccc}
\hline \hline$N$ & \multicolumn{3}{c}{$\sigma_{R}=0.8$} & & & $\sigma_{R}=2$ \\
& $\rho=0$ & $\rho=0.5$ & $\rho=1$ & $\rho=0$ & $\rho=0.5$ & $\rho=1$ \\
\hline 1 & 0.01653 & 0.01653 & 0.01653 & 0.07375 & 0.07375 & 0.07375 \\
2 & 0.01653 & 0.01435 & 0.00438 & 0.07375 & 0.06952 & 0.05251 \\
3 & 0.01653 & 0.01274 & 0.00281 & 0.07375 & 0.06647 & 0.04761 \\
4 & 0.01653 & 0.01156 & 0.00230 & 0.07375 & 0.06426 & 0.04550 \\
5 & 0.01653 & 0.01066 & 0.00205 & 0.07375 & 0.06257 & 0.04432 \\
\hline \hline
\end{tabular}

conditions. The number of relays has a stronger effect on the outage performance for higher values of $\rho$, i.e., when system leads to full-correlation. In the case of completely outdated PRS $(\rho=0)$, the information used for relay selection and actual CSI will be independent. In that case, it is not important which relay is selected and diversity gain is not accomplished. The results illustrate that the effect of the number of relays on outage probability is more pronounced in weaker turbulence conditions in FSO link.

The same effects can be concluded from results in Table I, which shows the values of outage probability for various numbers of relays when the correlation coefficient takes values: $\rho=0$ (completely outdated PRS), $\rho=0.5$ and $\rho=1$ (perfect PRS), with parameters $\gamma_{t h}=-10 \mathrm{~dB}, \mu_{1}=\mu_{2}=20 \mathrm{~dB}$ and $k=N$. When $N=1$, there will be no relay selection procedure. As it was mentioned, when $\rho=0$, the CSI used for relay selection is completely outdated so the increasing of $N$ will make no difference in the outage performance. When $\rho=1$ and $\sigma_{R}=0.8$, the outage performance is improved by $75.3 \%$ when PRS with two relays is implemented, compared to system with single relay; and $10.87 \%$ for improving the system with four to the system with five relays. Considering the expenses and difficulty of the implementing the PRS systems with greater number of relays, it may be concluded that the PRS systems with two relays are the most cost effective. Adopting $N=2$ and weak turbulence conditions, the system performance will be improved by $69.48 \%$ when $\rho$ is changed from 0.5 to 1 , while improvement of $24.47 \%$ is noticed in moderated conditions. When the FSO transmission is performed in favorable conditions, the influence of correlation has significant role in outage performance. Beside the complexity of the system referring to number of relays, it is interesting to analyse energy-efficiency of RF/FSO relaying systems in addition to outage probability and average BER performance [27], which will be a topic of our further work.

Fig. 4 shows the outage probability dependence on average SNR per RF link for different values of correlation coefficient and average electrical SNR per FSO link. Increasing of the correlation coefficient leads to the better system performance. First, numerical results are obtained assuming average electrical SNR per FSO link is constant, i.e., $\mu_{2}=25 \mathrm{~dB}$. It is observed that increment of $\mu_{1}$ leads to existence of outage floor, meaning that further increasing of average power of RF signal will not improve system performance. Furthermore, results are presented considering that $\mu_{2}$ simultaneously increases with $\mu_{1}$, which implies that the outage floor does not exist.

In Fig. 5, the average BER dependence on $\mu_{1}=\mu_{2}$ for two modulation schemes in various turbulence conditions is

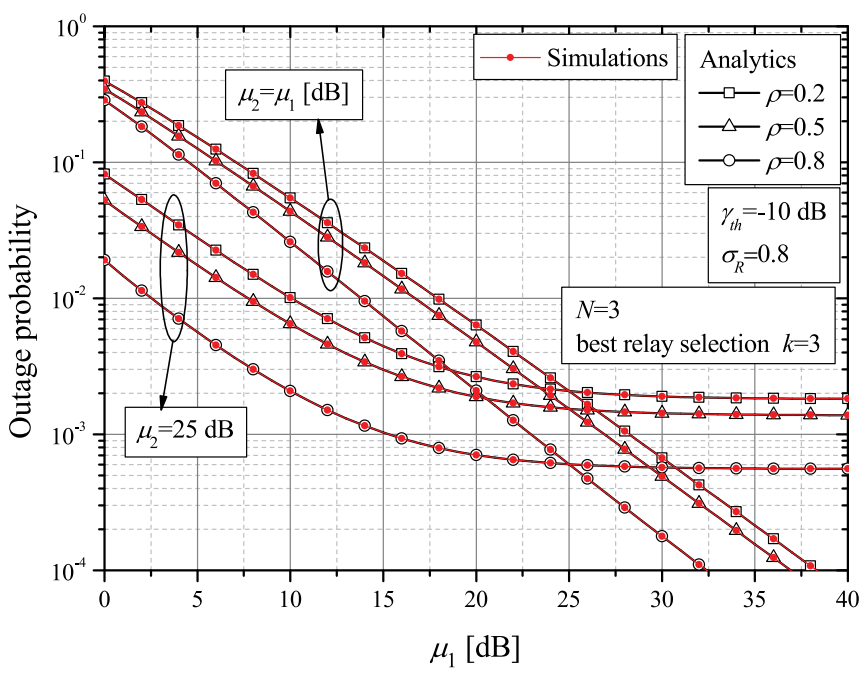

Fig. 4. Outage probability versus average SNR per RF link for different values of correlation coefficient and average electrical SNR per FSO link.

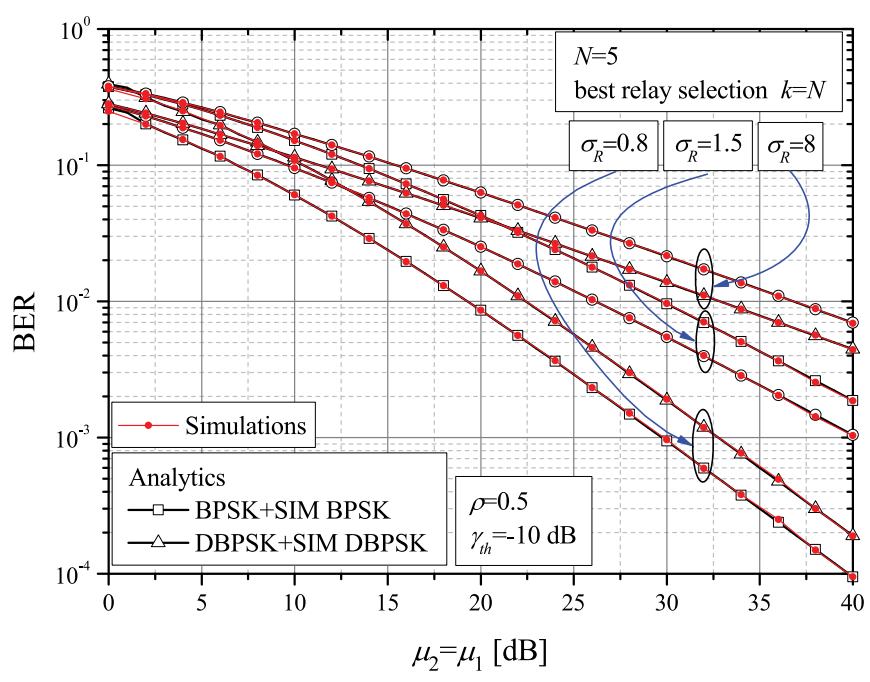

Fig. 5. Average BER versus $\mu_{1}=\mu_{2}$ for different types of binary modulation in different turbulence conditions.

presented. As it was expected, the modulation scheme 1, consisting of BPSK and SIM BPSK, shows better performance compared to scheme 2, consisting of DBPSK and SIM DBPSK. Also, the best average BER performance is manifested in weak turbulence conditions.

The average BER versus $\rho$ for different types of binary modulations is observed in Fig. 6. When the worst relay is selected, the increasing of correlation coefficient leads to worse system performance. If $\rho \rightarrow 0$, the estimated CSI used for PRS decision is completely outdated and there is no correlation with the current CSI, so it will not be important which relay is selected. For $\rho<0.1$, it is manifested by overlapping the appropriate curves relating to the best and worst relay selection.

\section{CONCLUSION}

In this paper, we have derived novel closed-form outage probability and average BER expressions of mixed dual-hop RF/FSO 


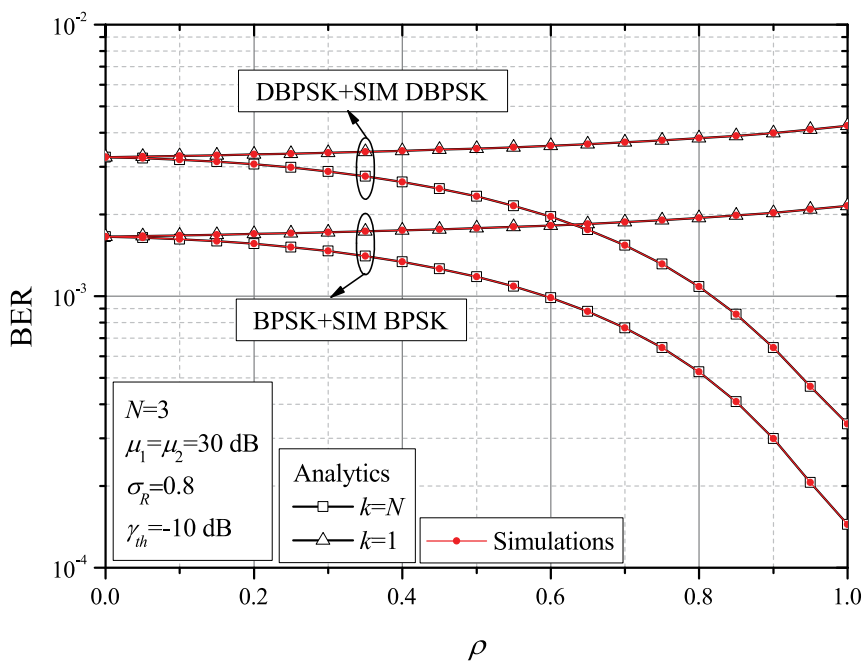

Fig. 6. Average BER versus correlation coefficient $\rho$ for different types of binary modulation.

relaying system with the semi-blind AF relaying under partial selection with outdated channel state estimation. With an assumption that the best relay is not necessarily able to forward the signal, more general analysis where PRS selects the $k$ th worst relay is adopted. The RF link is assumed to be Rayleigh fading channel, while the second FSO hop experiences GammaGamma atmospheric turbulence. The analytical results have been simplified to the case when PRS is performed based on perfect CSI. Also, considered system can be reduced to the one with single relay, so the derived expressions come to results already reported.

The effects of turbulence strength, average SNR over RF hop, average electrical SNR over FSO hop, number of relays, rank of selected relay and temporal correlation coefficient between source-relay channel and its outdated estimation have been observed. The results have illustrated that the system performance is dependent on the channel state of the second FSO hop to a large extent. The improvement of the outage probability and average BER performance with increasing the number of relays or the values of the correlation coefficient is more dominant in weak turbulence conditions over FSO link.

With respect to the expenses and difficulty of the implementing relaying systems with PRS, it is concluded that the RF/FSO systems employing two relays are the most cost effective. In addition to outage probability and average BER performance, analysis of complexity and energy-efficiency of RF/FSO relaying systems could be also performed, which will be a topic of our further investigation.

\section{APPENDIX A}

DERIVATION OF THE PDF OF THE INSTANTANEOUS SNR PER THE FIRST HOP $\gamma_{1(k)}$

The PRS is based on the outdated CSI of $S-R_{k}$ hop, so the PDF of $\tilde{\gamma}_{1(k)}$ is required for relay selection. By arranging $\tilde{\gamma}_{1(l)}$ for $l=1, \ldots, N$ in an increasing order of magnitude, the order statistics of $\tilde{\gamma}_{1(1)} \leq \tilde{\gamma}_{1(2)} \leq \cdots \leq \tilde{\gamma}_{1(k)} \leq \cdots \leq \tilde{\gamma}_{1(N)}$ is found [12].
The RF link experiences Rayleigh fading, so PDF and CDF of $\tilde{\gamma}_{1(l)}$ are given respectively as [12]

$$
f_{\tilde{\gamma}_{1(l)}}(y)=\frac{1}{\mu_{1}} e^{-\frac{y}{\mu_{1}}}, F_{\tilde{\gamma}_{1(l)}}(y)=1-e^{-\frac{y}{\mu_{1}}} .
$$

Next, $\gamma_{1(l)}$ and $\tilde{\gamma}_{1(l)}$ are two correlated exponentially distributed random variables. Their joint PDF is given as [16, eq. (9.296) for $m=1]$

$$
f_{\gamma_{1(l)}, \tilde{\gamma}_{1(l)}}(x, y)=\frac{1}{(1-\rho) \mu_{1}^{2}} e^{-\frac{x+y}{(1-\rho) \mu_{1}}} I_{0}\left(\frac{2 \sqrt{\rho x y}}{(1-\rho) \mu_{1}}\right),
$$

where $I_{\nu}(\cdot)$ represents the $\nu$ th order modified Bessel function of the first kind [21, eq. (8.406)].

The PDF of the instantaneous SNR $\gamma_{1(k)}$ per the first hop between $S$ and $R_{k}$ can be found as

$$
f_{\gamma_{1(k)}}(x)=\int_{0}^{\infty} f_{\gamma_{1(k)} \mid \tilde{\gamma}_{1(k)}}(x \mid y) f_{\tilde{\gamma}_{1(k)}}(y) d y,
$$

where $f_{\gamma_{1(k)} \mid \tilde{\gamma}_{1(k)}}(x \mid y)=f_{\gamma_{1(l)}, \tilde{\gamma}_{1(l)}}(x, y) / f_{\tilde{\gamma}_{1(l)}}(y)$ is the PDF of $\gamma_{1(k)}$ conditioned on $\tilde{\gamma}_{1(k)}$. The PDF of $\tilde{\gamma}_{1(k)}$ is given by [12]

$$
\begin{aligned}
f_{\tilde{\gamma}_{1(k)}}(y)= & \frac{N !}{(k-1) !(N-k) !} f_{\tilde{\gamma}_{1(l)}}(y) \\
& \times\left(F_{\tilde{\gamma}_{1(l)}}(y)\right)^{k-1}\left(1-F_{\tilde{\gamma}_{1(l)}}(y)\right)^{N-k} .
\end{aligned}
$$

After some mathematical manipulations, the PDF of $\gamma_{1(k)}$ is derived and expressed by (8).

\section{APPENDIX B}

\section{DERIVATION OF THE OUTAGE PROBABILITY}

The outage probability can be derived by substituting (9) and (11) into (14) leading to

$$
\begin{aligned}
P_{\text {out }}= & \frac{(\alpha \beta)^{\frac{\alpha+\beta}{2}}}{\Gamma(\alpha) \Gamma(\beta) \mu_{2}^{\frac{\alpha+\beta}{4}}} \int_{0}^{\infty} x^{\frac{\alpha+\beta}{4}-1} K_{\alpha-\beta}\left(2 \sqrt{\alpha \beta \sqrt{\frac{x}{\mu_{2}}}}\right) \\
& \times\left\{1-k\left(\begin{array}{c}
N \\
k
\end{array}\right) \sum_{n=0}^{k-1}\left(\begin{array}{c}
k-1 \\
n
\end{array}\right) \frac{(-1)^{n}}{(N-k+n+1)}\right. \\
& \left.\times e^{-\frac{(N-k+n+1) \gamma_{t h}\left(1+\frac{C}{x}\right)}{((N-k+n)(1-\rho)+1) \mu_{1}}}\right\} d x .
\end{aligned}
$$

Eq. (35) can be rewritten as

$$
\begin{aligned}
P_{\text {out }}= & 1-\frac{(\alpha \beta)^{\frac{\alpha+\beta}{2}}}{\Gamma(\alpha) \Gamma(\beta) \mu_{2}^{\frac{\alpha+\beta}{4}}} k\left(\begin{array}{c}
N \\
k
\end{array}\right) \sum_{n=0}^{k-1}\left(\begin{array}{c}
k-1 \\
n
\end{array}\right) \\
& \times \frac{(-1)^{n}}{(N-k+n+1)} e^{-\frac{(N-k+n+1) \gamma_{t h}}{((N-k+n)(1-\rho)+1) \mu_{1}}} \times I_{1},
\end{aligned}
$$

where $I_{1}$ is integral given by

$$
\begin{aligned}
I_{1}= & \int_{0}^{\infty} x^{\frac{\alpha+\beta}{4}-1} e^{-\frac{(N-k+n+1) \gamma_{t h} C}{((N-k+n)(1-\rho)+1) \mu_{1} x}} \\
& \times K_{\alpha-\beta}\left(2 \sqrt{\alpha \beta \sqrt{\frac{x}{\mu_{2}}}}\right) d x .
\end{aligned}
$$


The integral $I_{1}$ is solved in terms of Meijer's $G$ functions [21, eq. (9.301)] as follows. Using [25, eqs. (01.03.26.0004.01) and (07.34.16.0002.01)], the exponential function in (37) is expressed as

$$
\begin{aligned}
& e^{-\frac{(N-k+n+1) \gamma_{t h} C}{((N-k+n)(1-\rho)+1) \mu_{1} x}} \\
& \quad=G_{0,1}^{1,0}\left(\frac{(N-k+n+1) \gamma_{t h} C}{((N-k+n)(1-\rho)+1) \mu_{1} x} \mid \begin{array}{c}
- \\
0
\end{array}\right) \\
& \quad=G_{1,0}^{0,1}\left(\frac{((N-k+n)(1-\rho)+1) \mu_{1} x}{(N-k+n+1) \gamma_{t h} C} \mid \begin{array}{c}
1 \\
-
\end{array}\right),
\end{aligned}
$$

while the modified Bessel function of the second kind is presented using [25, eq. (03.04.26.0009.01)] as

$$
K_{\alpha-\beta}\left(2 \sqrt{\alpha \beta \sqrt{\frac{x}{\mu_{2}}}}\right)=\frac{1}{2} G_{0,2}^{2,0}\left(\alpha \beta \sqrt{\frac{x}{\mu_{2}}} \mid \begin{array}{l}
- \\
\kappa_{2}
\end{array}\right),
$$

where

$$
\kappa_{2}=\frac{\alpha-\beta}{2}, \quad \frac{\beta-\alpha}{2}, .
$$

Finally, integral $I_{1}$ is solved by [25, eq. (07.34.21.0013.01)] as

$$
\begin{aligned}
I_{1}= & \left(\frac{(N-k+n+1) \gamma_{t h} C}{((N-k+n)(1-\rho)+1) \mu_{1}}\right)^{\frac{\alpha+\beta}{4}} \\
& \times G_{0,5}^{5,0}\left(\frac{\alpha^{2} \beta^{2} \gamma_{t h} C(N-k+n+1)}{2^{4} \mu_{2} \mu_{1}((N-k+n)(1-\rho)+1)} \mid \begin{array}{c}
- \\
\kappa_{3}
\end{array}\right),
\end{aligned}
$$

where

$$
\kappa_{3}=\frac{\alpha-\beta}{4}, \quad \frac{\alpha-\beta+2}{4}, \quad \frac{\beta-\alpha}{4}, \quad \frac{\beta-\alpha+2}{4}, \quad-\frac{\alpha+\beta}{4} .
$$

Substituting (41) into (36) and applying [25, eq. (07.34.16.0001.01)], the novel analytical expression for the outage probability is derived in the form of (15).

\section{ACKNOWLEDGMENT}

The authors would like to thank the Associate Editor and reviewers for many valuable comments and discussions that considerably improved the quality of the manuscript.

\section{REFERENCES}

[1] E. Lee, J. Park, D. Han, and G. Yoon, "Performance analysis of the asymmetric dual-hop relay transmission with mixed RF/FSO links," IEEE Photon. Technol. Lett., vol. 23, no. 21, pp. 1642-1644, Nov. 2011.

[2] H. Samimi and M. Uysal, "End-to-end performance of mixed RF/FSO transmission systems," IEEE/OSA J. Opt. Commun. Netw., vol. 5, no. 11, pp. 1139-1144, Nov. 2013.

[3] I. S. Ansari, F. Yilmaz, and M.-S. Alouini, "On the performance of mixed RF/FSO dual-hop transmission systems," in Proc. IEEE 77th Veh. Technol. Conf., Jun. 2013, pp. 1-5.

[4] I. S. Ansari, F. Yilmaz, and M.-S. Alouini, "Impact of pointing errors on the performance of mixed RF/FSO dual-hop transmission systems," IEEE Wireless Commun. Lett., vol. 2, no. 3, pp. 351-354, Jun. 2013.

[5] I. S. Ansari, M.-S. Alouini, and F. Yilmaz, "On the performance of hybrid RF and RF/FSO fixed gain dual-hop transmission systems," in Proc. Saudi Int. Electron., Commun. Photon. Conf., Apr. 2013, pp. 1-6.

[6] I. S. Ansari, F. Yilmaz, and M.-S. Alouini, "On the performance of mixed RF/FSO variable gain dual-hop transmission systems with pointing errors," in Proc. IEEE 78th Veh. Technol. Conf., Sep. 2013, pp. 1-5.
[7] I. S. Ansari, F. Yilmaz, and M.-S. Alouini, "On the performance of hybrid RF and RF/FSO dual-hop transmission systems," in Proc.2nd Int. Workshop Opt. Wireless Commun., Newcastle Upon Tyne, U.K., Oct. 2013, pp. 45-49.

[8] N. D. Chatzidiamantis, D. S. Michalopoulos, E. E. Kriezis G. K. Karagiannidis, and R. Schober, "Relay selection protocols for relayassisted free-space optical systems," IEEE/OSA J. Opt. Commun. Netw. vol. 5, no. 1, pp. 92-103, Jan. 2013.

[9] I. Krikidis, J. Thompson, S. Mclaughlin, and N. Goertz, "Amplify-andforward with partial relay selection," IEEE Commun. Lett., vol. 12, no. 4, pp. 235-237, Apr. 2008.

[10] D. B. da Costa and S. Aïssa, "End-to-end performance of dual-hop semiblind relaying systems with partial relay selection," IEEE Trans. Wireless Commun., vol. 8, no. 8, pp. 4306-4315, Aug. 2009.

[11] H. A. Suraweera, D. S. Michalopoulos, and G. K. Karagiannidis, "Semiblind amplify-and-forward with partial relay selection," Electron. Lett. vol. 45, no. 6, pp. 317-319, Mar. 2009.

[12] H. A. Suraweera, M. Soysa, C. Tellambura, and H. K. Garg, "Performance analysis of partial relay selection with feedback delay," IEEE Signal Process. Lett., vol. 17, no. 6, pp. 531-534, Jun. 2010.

[13] M. Soysa, H. A. Suraweera, C. Tellambura, and H. K. Garg, "Partial and opportunistic relay selection with outdated channel estimates," IEEE Trans. Commun., vol. 60, no. 3, pp. 840-850, Mar. 2012.

[14] D. S. Michalopoulos, H. A. Suraweera, G. K. Karagiannidis, and R. Schober, "Amplify-and-forward relay selection with outdated channel estimates," IEEE Trans. Commun., vol. 60, no. 5, pp. 1278-1290, May 2012.

[15] N. S. Ferdinand, N. Rajatheva, and M. Latva-aho, "Effects of feedback delay in partial relay selection over Nakagami- $m$ fading channels," IEEE Trans. Veh. Technol., vol. 61, no. 4, pp. 1620-1634, May 2012.

[16] M. K. Simon and M.-S. Alouni, Digital Communication Over Fading Channels, 2nd ed. New York, NY, USA: Wiley, 2004.

[17] L. C. Andrews and R. N. Philips, Laser Beam Propagation Through Random Media, 2nd ed. Bellingham, WA, USA: SPIE, 2005.

[18] Z. Ghassemlooy, W. Popoola, and S. Rajbhandari, Optical Wireless Communications: System and Channel Modelling With MATLAB. Boca Raton, FL, USA: CRC Press, 2013.

[19] S. Arnon, J. Barry, G. Karagiannidis, R. Schober, and M. Uysal, Eds.,Advanced Optical Wireless Communication Systems. New York, NY, USA, USA: Cambridge Univ. Press, 2012.

[20] M. Niu, J. Cheng, and J. F. Holzman, "Error rate performance comparison of coherent and subcarrier intensity modulated optical wireless communications," IEEE/OSA J. Opt. Commun. Netw., vol. 5, no. 6, pp. 554-564, Jun. 2013.

[21] I. S. Gradshteyn and I. M. Ryzhik, Table of Integrals, Series, and Products., 6th ed. New York, NY, USA: Academic, 2000.

[22] N. D. Chatzidiamantis, A. S. Lioumpas, G. K. Karagiannidis, and S. Arnon, "Optical wireless communications with adaptive subcarrier PSK intensity modulation," in Proc. IEEE Global Telecommun. Conf., Dec. 2010, pp. 1-16.

[23] A. A. Farid, and S. Hranilovic, "Outage capacity optimization for freespace optical links with pointing errors," J. Lightw. Technol., vol. 25, no. 7, pp. 1702-11710, Jul. 2007.

[24] E. J. Lee, and V. W. S. Chan, "Part 1: Optical communication over the clear turbulent atmospheric channel using diversity," IEEE J. Sel. Areas Commun., vol. 22, no. 9, pp. 1896-11906, Nov. 2004.

[25] The Wolfarm Functions Site. (2008). [Online] Available: http:/ functions.wolfarm.com

[26] I. S. Ansari, S. Al-Ahmadi, F. Yilmaz, M.-S. Alouini, and H. Yanikomeroglu, "A new formula for the BER of binary modulations with dual-branch selection over generalized-K composite fading channels," IEEE Trans. Commun., vol. 59, no. 10, pp. 2654-2658, Oct. 2011.

[27] A. Zafar, R. M. Radaydeh, Y. Chen, and M.-S. Alouini, "Power allocation strategies for fixed-gain half-duplex amplify-and-forward relaying in Nakagami-m fading," IEEE Trans. Wireless Commun., vol. 13, no. 1, pp. 159-173, Jan. 2014

Authors' biographies not available at the time of publication. 